\title{
A device for silent on-line recording of continuously changing behavioral measures
}

\author{
PETER L. ROGERS \\ Harvard School of Public Health, Boston, Massachusetts 02115 \\ JOHN GOLENSKI \\ Harvard Graduate School of Education, Cambridge, Massachusetts 02138 \\ and \\ LEE WILLIAMS \\ Massachusetts Institute of Technology, Cambridge, Massachusetts 02139
}

\begin{abstract}
A nonintrusive method of monitoring continuously changing behavior frequently is required in experimentation with human subjects, particularly infants. The touch-sensor switch box provides the observer with an ongoing record on a polygraph and allows the observer to give undivided attention to the subject. The mode of operation is completely silent.
\end{abstract}

Since behavioral state has proved to be a significant interacting variable in infant discrimination research (Ashton, 1971; Clifton \& Nelson, 1976; Prechtl, 1974), continuous monitoring of the ongoing state of the infant is advisable during experimental sessions. Two methods are traditionally used: (1) physiological measures (e.g., EKG, heart rate, etc.) are recorded by a polygraph simultaneously with other behavioral events that are linked to experimental treatments, and (2) observational measures (which typically involve coding infant state in arbitrary time periods) are written on a data sheet by an observer.

Although physiological measures have the advantage of providing a continuous on-line record, they obviously require intrusive instrumentation which could, itself, affect the infant's state. On the other hand, observations recorded with paper and pencil require transcribing state judgments that may divert the observer's attention from the infant, at least momentarily, during the recording time period. Measuring infant state with on-line event recorders usually involves hardware that can create enough noise to alter state.

The touch-sensor switch box provides the observer with a method of continuously monitoring an infant subject's state without auditory feedback to the infant or the use of contact recording devices such as electrodes or strain gauges. In addition, the mode of operation allows both continuous recording by a polygraph or some other mechanical device and uninterrupted attention from the observer. Although this device was de-

This work was supported by a research fellowship granted by the National Institutes of Health, Division of Child Health and Human Development (1 F22 HC0 1912-01), and by a small grant awarded by the National Institute of Mental Health (1 R03 MH-27636-01). signed to provide a record of infant state, it can be used to advantage in any experimental situation in which an on-line record of observed behavioral changes is needed. It is silent, easy to operate, and economical.

\section{DESIGN CONCEPT}

The touch-sensor switch box is designed to provide the experimenter-observer with five discrete dc voltage levels that can drive one channel of a polygraph. Pre. viously, this has been accomplished by using a battery or other direct-current voltage source, a series string of resistors arranged as a voltage divider connected across this source, and a number of toggle switches or pushbuttons to select different voltage levels. The new circuit employs a voltage divider, but substitutes completely silent, electronic circuitry for the switches or pushbuttons. Appropriate voltage levels to be fed to the polygraph are selected by using different fingers of one hand to complete the circuit between two metal touchsensor contacts. The low-voltage high-impedance circuit presents no shock hazard.

The output of the prototype touch-sensor switch box was connected to the direct-drive input of the driver amplifier of a Grass Model 7 polygraph. The switch box supply leads were connected to the $+12-\mathrm{V}$ dc regulated output terminals on the rear apron of the polygraph's main power supply (red lead to $+12 \mathrm{~V}$, white lead to chassis ground).

\section{CIRCUIT DESCRIPTION}

The voltage divider portion of the circuit consists of fixed resistors R1 through R5 and variable resistor R6 (see Figure 1). The latter can be either a standard-size linear potentiometer or a small carbon trimmer. Re- 


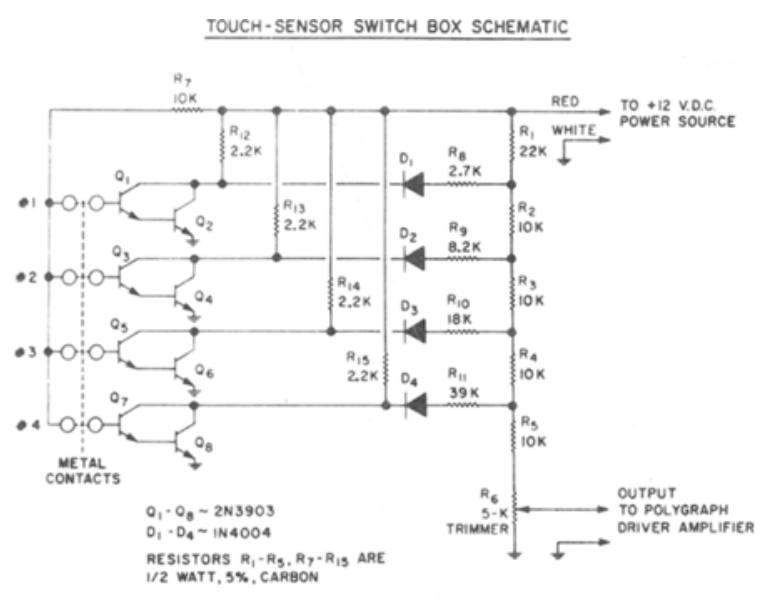

Figure 1. Touch-sensor switch box schematic.

sistors R8 through R11 provide approximately $.2 \mathrm{~V}$ difference between consecutive voltage levels. These resistor values ( $R 8$ through $R 11$ ) may be changed slightly to provide specific output voltages as required. Some interaction may be expected; changing one value may necessitate changing the other three resistors as well. Diodes D1 through D4 isolate the transistor switches from the voltage divider and guarantee reliable operation. Each transistor switch consists of two silicon NPN transistors connected in a Darlington configuration. Resistors R12 through R15 are the collector loads for each pair of switching transistors. With all touch-sensor contacts unbridged, the transistor switches are "off" and the output to the polygraph is $1.0 \mathrm{~V}$. (Following construction, variable resistor R6 is adjusted to give an output of $1.0 \mathrm{~V}$, as measured on a voltmeter, with all touch-sensor contacts open or unbridged.) When a pair of contacts (one common and one base connected) is bridged with the finger, the associated Darlington pair of transistors is forward biased and current flows through the pair's collector load resistor. The cathode end of the diode, connected to the pair, is pulled down toward ground, or $0 \mathrm{~V}$, and a corresponding voltage drop appears across the resistor (R8, R9, R10, or R11) connected between the diode and the voltage divider resistor string. The effect is to alter the resistance ratio of the voltage divider, changing the output voltage, and causing the polygraph pen to move to a new position. When the finger is removed, the pen returns to its original position. Due to the different connection points along the voltage divider, and the different values of resistors R8, R9, R10, and R11, four entirely separate and discrete pen position shifts are possible.

\section{CONSTRUCTION}

The components may be mounted on terminal strips or on a small circuit board. We used a $2 \times 4$ in. piece of $.1 \times .1$ in. Veroboard (Vero Electronics, Inc., 171 Bridge Road, Hauppauge, New York 11787) for the prototype. Care should be taken not to overheat the diodes or transistors. Power supply leads should be color coded, red for $+12 \mathrm{~V}$ and white for ground.

Chrome-plated upholstery tacks may be used as touch-sensor contacts. Four contacts are mounted (by drilling small holes $7 / 16$ in. off the longitudinal center line) in a row on the cover of a phenolic meter case with $11 / 2$-in. space between them. The metal contacts may be mounted in any material that is a good insulator. Each of the four contacts is connected to the base of one of the transistors Q1, Q3, Q5, and Q7. Four additional contacts are mounted in parallel with those in the first row at a distance of approximately $7 / 8$ in. (see Figure 2). The second group of four contacts are connected together in common and wired to one end of $\mathrm{R} 7$, a current-limiting resistor.

In order not to load the circuit too heavily, the input impedance of the polygraph amplifier or other recording device must be reasonably high, that is, on the order of $10,000 \mathrm{ohms}$ or more. One solution to this problem is to connect one of the new CDA (currentdifferencing amplifier) operational amplifiers to the output to serve as a simple noninverting buffer. The MC3401P by Motorola or LM3900N by National are good choices. These CDAs operate on a single-ended supply and can be used for digital as well as linear applications. By buffering the output, the touchsensor switch box can be more easily interfaced with a variety of recording equipment, such as a voltage-tofrequency converter coupled with an audio tape recorder. Data gathered in this manner can then be fed into a computer via an analog-to-digital converter and analyzed, using appropriate software.

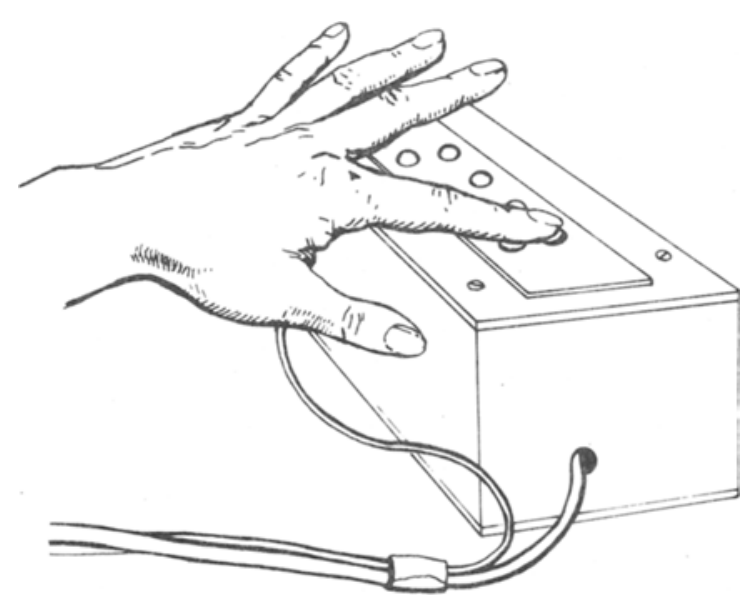

Figure 2. Touch sensor switch box showing position of metal contacts. 


\section{REFERENCES}

Ashron. R. The effects of environment upon state cycles in the human newborn. Journal of Experimental Child Psychology, 1971, 2, 1-9.

Clifton, R. K., \& Nelson, M. N. Developmental study of habituation in infants: The importance of paradigm, response system. and state. In T. J. Tighe \& R. N. Leaton (Eds.), Habituation: Perspectives from child development, animal behavior. and neurophysiology. Hillsdale, N.J: Lawrence Erlbaum, 1976. Pp. 159-205.

Prechti, H. F. R. The behavioral states of the newborn infant (A review), Brain Research, 1974, 76, 185-212.

(Received for publication October 4, 19\%7; revision accepted December 29, 1977.) 